\title{
Zurek-Kibble domain structures: The Dynamics of Spontaneous Vortex formation in Annular Josephson Tunnel Junctions
}

\author{
R. Monaco ${ }^{a *}$, J. Mygind $^{b \dagger}$ and R. J. Rivers ${ }^{c \dagger}$ \\ a) Istituto di Cibernetica del C.N.R., I-80078, Pozzuoli, Italy \\ and Unita' INFM-Dipartimento di Fisica, Universita' di Salerno, \\ I-84081 Baronissi, Italy \\ b) Department of Physics, Technical University of Denmark, \\ B309, DK-2800 Lyngby, Denmark \\ c) U.K. Centre of Theoretical Physics, University of Sussex, \\ Brighton, BN1 9QJ, U.K.
}

(October 26, 2018)

Phase transitions create a domain structure with defects, that has been argued by Zurek and Kibble to depend in a characteristic way on the quench rate. In this letter we present an experiment to measure the ZK scaling exponent $\sigma$. Using long symmetric Josephson Tunnel Junctions, for which the predicted index is $\sigma=0.25$, we find $\sigma=0.27 \pm 0.05$. Further, we agree with the ZK prediction for the overall normalisation.

PACS Numbers : 11.27.+d, 05.70.Fh, 11.10.Wx, 67.40.Vs

Because phase transitions take place in a finite time, causality guarantees that correlation lengths remain finite. Order parameter fields become frustrated, and defects arise so as to mediate the correlated regions with different ground states. Since defects are, in principle, observable, they provide an excellent experimental signature for the way in which transitions are implemented.

For condensed matter systems, which include the long annular Josephson Tunnel Junctions (JTJs) that we shall discuss below, Zurek [1]:2] suggested that causality alone is sufficient to determine the initial density of defects arising in a continuous transition. In this he paralleled proposals made by Kibble [3] in the context of quantum field theory models of the early universe.

As applied to JTJs, the idea is as follows. Consider a thin linear JTJ with critical temperature $T_{c}$, cooled through that temperature so that, if $T(t)$ is the temperature at time $t$, then $T(0)=T_{c} . \dot{T}(0)=-T_{c} / \tau_{Q}$ defines the quench time $\tau_{Q}$. Suppose, at time $t$, that $c(t)=c(T(t))$ is the Swihart velocity 国, vanishing at $t=0$, and that $\xi_{a d}(t)=\xi_{a d}(T(t))$ is the adiabatic healing length (the Josephson length $\lambda_{J}(T(t))$ ), diverging at $t=0$. The first time that fluxons (or Josephson vortices), the defects of linear JTJs, can appear is at time $\bar{t}$, when $\left|\dot{\xi}_{a d}(t)\right| \simeq c(t)$.

For the case in hand, $\bar{t}$ has the form $\bar{t}=\sqrt{\tau_{Q} \tau_{0}}$, where $\tau_{0} \ll \tau_{Q}$ is the relaxation time of the longest wavelength modes. Details are given in papers [6,7] by two

\footnotetext{
${ }^{*}$ E-mail: roberto@sa.infn.it

${ }^{\dagger}$ E-mail: myg@fysik.dtu.dk

${ }^{\ddagger}$ E-mail: r.rivers@ic.ac.uk Permanent address: Blackett Laboratory, Imperial College, London, SW7 2BZ, U.K.
}

of us (R.M and R.R). As a result, $\tau_{Q} \gg \bar{t} \gg \tau_{0}$. If $\xi_{a d}(t) \sim \xi_{0}\left(t / \tau_{Q}\right)^{-\nu}$ for $t \sim 0$, where $\xi_{0}$ is simply related to $\xi_{a d}(T=0)$, the cold fluxon size, then the initial domain size and fluxon separation is predicted to be

$$
\bar{\xi} \sim \xi_{a d}(\bar{t})=\xi_{0}\left(\frac{\tau_{Q}}{\tau_{0}}\right)^{\sigma} \gg \xi_{0},
$$

where $\sigma=\nu / 2$. We term $\sigma$ the Zurek-Kibble (ZK) characteristic index.

The arguments are not specific to JTJs. Prior to our experiment, five other condensed matter experiments had been performed to test the prediction (11) for the separation of defects at their production, two experiments [8.9] on superfluid ${ }^{3} \mathrm{He}$, two [10,11] on superfluid ${ }^{4} \mathrm{He}$, and one 12 on high temperature superconductors (HTSC). In addition, an experiment 13 on JTJs by two of us (R.M and J.M) was compatible with (11), although it had not been performed with a test of (11) in mind.

Before discussing our new experiment a few comments are in order. The experiments [8,9] on superfluid ${ }^{3} \mathrm{He}-\mathrm{B}$ rely on the fact that, when it is bombarded with slow neutrons, $n+{ }^{3} \mathrm{He} \rightarrow p+{ }^{3} H+760 \mathrm{keV}$. The energy released in such a collision leads to a hot spot which, when cooled by its environment below $T_{c}$, leaves behind a tangle of vortices (the topological defects in this system). $\tau_{Q}$ is fixed by the nuclear process that breaks up the ${ }^{3} \mathrm{He}$ atom. With only a single data point conflating both normalisation and $\sigma$ it is not possible to confirm the predicted value $\sigma=1 / 4$. However, both experiments are highly compatible with (11), agreeing to a factor of a few in each case.

In principle, the ${ }^{4} \mathrm{He}$ experiments [10,11], which use a pressure quench with a varying timescale $\tau_{Q}$ to implement the transition, allow for a more complete test. Yet again, vortices are the relevant defects. In practice, the most reliable experiment [11] sees no vortices. This is not necessarily a sign of failure in that it has been suggested [14] that the vortices decay too fast to be seen. This is irrespective [15] of whether a pressure quench, which preserves high thermal fluctuations, would of itself lead to somewhat different predictions. In this context, the vortices seen in an earlier ${ }^{4} \mathrm{He}$ experiment [10] were most likely an artefact of the experimental setup. 
The fifth experiment 12], on high- $T_{C}$ superconductors, measures total flux through a surface carried by the Abrikosov vortices. The vortex separation of (11) can be converted into a prediction for the flux, but no flux is seen in contradiction with this prediction, despite the phase separation that leads to the result being demonstrated elsewhere [16]. In this case there is no obvious explanation of the null result, despite later work [17 that takes the effect of gauge fields into account fully.

In summary, these early experiments (including [13]) have either provided one data point for (11), or have been null. Subsequently, two experiments of a very different type have been performed that permit varying quench rates and so an estimate for $\sigma$. The most recent [18] involves the Bénard-Marangoni conductionconvection transition. The defects here are not associated with the line zeroes of an order parameter field, and the viscosity-dependent $\sigma$ does not match the ZK prediction, most likely for that reason. The more relevant experiment [19] is carried out in a non-linear optical system, with complex beam-phase the order parameter. Increasing the light intensity (the control parameter in this case) leads to pattern formation (defects) at a critical value. The predicted $\sigma=1 / 4$ is recovered to good accuracy, but agreement with normalisation is not stated.

Our experiment, whose methodology and results we outline below, is also one in which, by varying $\tau_{Q}$, we can measure and compare $\sigma$ with its theoretical value, as well as confirming overall scale.

In its essence, we quench a long annular JTJ through its critical temperature and count such fluxons or Josephson vortices as appear. In its idealised form the annular JTJ consists of two dimensionally identical annuli of superconductors of narrow width, superimposed upon one another, separated by an insulating barrier in the same plane. In practice, it is sufficient for the lower superconductor to be a planar substrate upon which the other annulus sits. The effective theory for fluxons in JTJs [5] is the sine-Gordon model with respect to the field $\phi=\phi_{1}-\phi_{2}$, the difference in the phases of the complex order parameter fields in the separate superconductors. The Josephson vortices are then the sine-Gordon kinks.

The JTJs in our experiment are symmetric, by which is meant that the electrodes are made of identical superconducting material with the same energy gaps and the same $T_{c}$. This is confirmed by seeing that a) there is no logarithmic singularity in the voltage-current characteristic at finite voltages and b) the temperature dependence of the critical current is linear as $T$ approaches $T_{c}$. The outcome is that [6,7] $\nu=1 / 2$. Therefore, at the time of their formation the separation of fluxons is expected to be given by (1) with $\sigma=1 / 4$.

In terms of the parameters of the JTJs, the Josephson length at temperature $T$ is [5]

$$
\xi_{a d}(T)=\lambda_{J}(T)=\sqrt{\frac{\hbar}{2 e \mu_{0} d_{s} J_{c}(T)}},
$$

where $J_{c}(T)$ is the critical Josephson current at temperature $T$. Typically $\lambda_{J}(0)$ is in the $10-100 \mu m$ range and was equal to $7 \mu \mathrm{m}$ for the sample discussed below. In Ref. [7] $\xi_{0}$ has been inferred as

$$
\xi_{0}=\sqrt{\frac{\hbar}{2 e \mu_{0} d_{s} \alpha J_{c}(0)}} .
$$

The parameter $3 \lesssim \alpha \lesssim 5$ is given in terms of the superconductor gap energy $\Delta(T)$ and $T_{c}$. As for $\tau_{0}$, it is given as $\tau_{0}=\xi_{0} / c_{0}$, where $c_{0}$ defines the behavior $c(t)=c_{0}\left(t / \tau_{Q}\right)^{1 / 2}$ of the Swihart velocity for the system near $T=T_{c}$. If the thickness of the two superconducting electrodes differs, the effective thickness $d_{s}$ is the harmonic mean of the individual thicknesses [5].

Our samples are high quality, $500 \mu \mathrm{m}$ long, $3 \mu \mathrm{m}$ wide, $\mathrm{Nb} / \mathrm{Al}-A l_{o x} / \mathrm{Nb}$ JTJs fabricated on $0.5 \mathrm{~mm}$ thick silicon substrates using the trilayer technique (SNEAP) in which the junction is realized in the window opened in a $\mathrm{SiO}$ insulator layer. Details of the fabrication process can be found in Ref. 20]. For all samples the high quality has been inferred by a measure of the I-V characteristic at $T=4.2 \mathrm{~K}$. In fact, the subgap current $I_{s g}$ at $2 \mathrm{mV}$ was small compared to the current rise $\Delta I_{g}$ in the quasiparticle current at the gap voltage $V_{g}$, typically $\Delta I_{g}>35 I_{s g}$. The gap voltage was as large as $V_{g}=2.76 \mathrm{mV}$ and the maximum critical current $I_{c}$ was larger than $0.55 \Delta I_{g}$ for the overlap type junction. Furthermore, the application of a strong enough external magnetic field in the barrier plane completely suppressed any Josephson structures indicating the absence of electrical shorts in the barrier. At an order of magnitude level, we have $\tau_{0} \sim 0.1 \mathrm{ps}$ and $\xi_{0} \sim 1 \mu \mathrm{m}$. As a result $\bar{\xi} \sim 1 \mathrm{~mm}$ for $\tau_{Q} \sim 1 \mathrm{~s}$. We will be more specific later.

Our JTJs have a critical temperature $T_{c}=8.95 \mathrm{~K}$, whereas the individual superconductors have a critical temperature of $9.1 K$. Even at our fastest quench the conductors are superconducting, by which is meant that the Cooper-pair order parameter field has achieved its final magnitude, $1 \mathrm{~ms}$ before the JTJ can develop fluxons. This is necessary for (1) to be valid without modification [6,7], since only then is $\phi$ the relevant order parameter.

In order to vary the quenching time over the widest possible range, we have realized the experimental setup shown schematically in Fig.1. The annular JTJ samples are fabricated on a chip (shown endways on), mounted to a $C u$ block by a thermally insulating teflon sheet. The entire system is enclosed in a vacuum-tight can immersed in the liquid $H e$ bath. In all cases the heat is removed from the system by $H e$ exchange gas, using a manual pump. By varying the pressure of the gas we can modify the rate of cooling of the sample. On the other side of the block is a $50 \Omega$ carbon resistor, that enables us to heat and cool the JTJs on a relatively long timescale $\left(\tau_{Q}\right.$ from about 1 to $\left.10 \mathrm{~s}\right)$ depending on the He exchange gas pressure inside the can. With $\sigma=1 / 4$, we need to vary $\tau_{Q}$ by at least two orders of magnitude. This is not possible just by heating the block alone (even if it 
were smaller). To extend the range of $\tau_{Q}$ we mounted a small, pulse-driven, surface $100 \Omega$ resistor on the same side. This permits much smaller thermal cycles $(0.07$ to $0.2 s)$. These two completely different quenching techniques provide timescale ranges that do not overlap, leaving a gap between 0.2 and $1 s$, that would require a third quenching technique to be filled.

The whole system is then enclosed in a $\mu$-metal shielded cryostat. The temperature of the JTJ is monitored by measuring the junction gap voltage, which is proportional to the known superconductor gap energy $\Delta(T(t))$. All quenches were taken from $10 K$ to $4.2 K$ through $T_{c}=8.95 \mathrm{~K}$. By making use of the Thouless equation 21], it is possible to infer the temperature of the JTJ from the gap voltage in the range $8.2 \mathrm{~K}$ to $4.2 \mathrm{~K}$, extremely accurately at the upper end of the range, and with fluctuations of a few per cent at the lower. Whether for slow or fast cooling an excellent fit to the temperature of the JTJ in this range, for initial temperature $T_{i n}=10 \mathrm{~K}$ and final temperature $T_{\text {fin }}=4.2 \mathrm{~K}$, is given by the thermal relaxation equation

$$
T(t)=T_{i n}+\left(T_{i n}-T_{f i n}\right) e^{-\left(t-t_{0}\right) / \tau},
$$

where $\tau$ is the relaxation time which sets the cooling timescale. See Fig.2 for an example. This equation is then used to extrapolate $d T(t) / d t$ to the vicinity of $T_{C}$ and yields $\tau_{Q} \simeq 1.7 \tau$.

On cooling the system in this way, we expect fluxons to appear from the inhomogeneity of $\phi$ at the transition, according to (1). In the absence of any current through the barrier or applied external magnetic field the fluxons are in indifferent equilibrium as far as the barrier is homogeneous and pin-hole free. (In reality, there is a small pinning effect so that, after a short transient, the fluxons are static.) To make them visible, and countable, we apply a bias current, whereby they move as magnetic dipoles under the resulting Lorentz force, at speed less than the Swihart velocity. According to Josephson theory $N$ travelling fluxons (and antifluxons) with speed $v$ develop a voltage $V=N \Phi_{0} v / C$ across the junction, where $\Phi_{0}=h / 2 e$ is the flux in a (Josephson) fluxon and $C$ is the annulus circumference. This voltage can be measured, and the fluxon number determined. A detailed description of the experiment and the data will be given elsewhere 22].

Many samples have been measured, but only one had such a large critical current density (and sufficiently small $\lambda_{J}(T)$ ) that only 3000 thermal cycles were enough to get reliable statistics, and it is this sample that we shall discuss now. However, we stress that, within the less good statistics of the other samples, none gave results that were incompatible with (1).

The symmetric annular JTJ with which the experiment was performed had a circumference $C=500 \mu \mathrm{m}$, and width $\Delta r=4 \mu \mathrm{m}$. The effective superconductor thickness was $d_{s} \approx 250 \mathrm{~nm}$. At the final temperature $T_{\text {fin }}=4.2 \mathrm{~K}$, the critical current density was $J_{c}\left(T_{f i n}\right)=$
$3050 \mathrm{~A} / \mathrm{cm}^{2}$, the quality factor was $V_{m}=49 \mathrm{mV}$ and the Josephson length was $\lambda_{J}\left(T_{\text {fin }}\right)=6.9 \mu \mathrm{m}$. The velocity $c_{0}$ is calculated to be $c_{0} \approx 2.2 \times 10^{7} \mathrm{~m} / \mathrm{s}$. From this, we infer that $\xi_{0} \approx 3.8 \mu \mathrm{m}$ and $\tau_{0} \approx 0.17 \mathrm{ps}$. It follows that $\bar{\xi} \approx 5.9 \mathrm{~mm}$ for $\tau_{Q}=1 \mathrm{~s}$, several times the circumference of the JTJ.

As a result, the likelihood of finding a single fluxon is small. We estimate the probability of finding a fluxon in a single quench to be

$$
P_{1} \simeq \frac{C}{\bar{\xi}}=\frac{C}{\xi_{0}}\left(\frac{\tau_{Q}}{\tau_{0}}\right)^{-\sigma},
$$

where, from (11), $\sigma=0.25$.

In Fig. 3 we show a log-log plot for the measured $P_{1}$ as a function of $\tau_{Q}$, changed by varying the exchange gas pressure and by using both the fast and slow quenching techniques. Each data point corresponds to 300 quenches. Although the data does not distinguish between a single fluxon and a fluxon plus an antifluxon pair, the likelihood of the latter is sufficiently small that it can be ignored. Similarly, the data does not distinguish between no fluxons and a fluxon-antifluxon pair, with similar errors. We observe that the points are quite scattered, meaning that the data are statistically poor. Further, for the reasons given earlier there is a gap between fast and slow quenches, with the possibility for systematic error. Nonetheless, we have clear evidence that i) the trapping of a fluxon occurs on a purely statistical basis for identical conditions of each thermal cycle and ii) the probability to trap one fluxon is larger when the transition is performed at a faster speed (smaller quenching time) in accordance with the causality principle. This complements our qualitative results from other samples with smaller $J_{c}(0)$ (for which the statistics is too poor to permit a fit to (11)) that, at fixed $\tau_{Q}$, the probability of finding a fluxon decreases with increasing $\xi_{0}$.

Regardless of the data spread, to test (3) we attempted to fit the data with an allometric function $P_{1}=a \tau_{Q}^{-b}$, with $a$ and $b$ being free fitting parameters. We found, for the coefficient $a$, the best fitting value of $0.1 \pm 10 \%$ (taking $\tau_{Q}$ in seconds). This is in excellent agreement with the predicted value of $C \tau_{0}^{1 / 4} / \xi_{0} \approx 0.08 s^{1 / 4}$, given the fact that we only expect agreement in overall normalization to somewhat better than an order of magnitude. After the failure of the experiments discussed in 10 to find (reliable) defects at expected densities, if at all, this experiment shows that the ZK estimate remains sensible. Further, the best fitting curve, shown by the solid line in Fig.3, has a slope $b=0.27 \pm 0.05$, in remarkable agreement with its predicted value of 0.25 .

The ZK scenario needs further testing with JTJs for which there is a greater likelihood of observing fluxons. In [6] and [7] we observed that this is the case for significantly non-symmetric JTJs, for which the value of $\sigma$ is $\sigma=1 / 7$; a further experiment, with markedly nonsymmetric JTJs, is being planned. 
The authors thank L. Filippenko for the sample fabrication and V.P. Koshelets for usefull discussions. R.R. thanks the University of Salerno for hospitality. This work is, in part, supported by the COSLAB programme of the European Science Foundation, the Danish Research Council, and the Hartmann Foundation.

[1] W.H. Zurek, Nature 317, 505 (1985), Acta Physica Polonica B24, 1301 (1993).

[2] W.H. Zurek, Physics Reports 276, Number 4, Nov. 1996.

[3] T.W.B. Kibble, in Common Trends in Particle and Condensed Matter Physics, Physics Reports 67, 183 (1980).

[4] J.C. Swihart, J. Appl. Phys., 32, 461 (1961).

[5] A. Barone and G. Paterno', Physics and Applications of the Josephson Effect, John Wiley \& Sons, New York (1982).

[6] E. Kavoussanaki, R. Monaco and R.J. Rivers, Phys. Rev. Lett. 85, 3452 (2000).

[7] R. Monaco, R.J. Rivers and E. Kavoussanaki, Journal of Low Temperature Physics 124, 85 (2001).

[8] C. Bauerle et al., Nature 382, 332 (1996).

[9] V.M.H. Ruutu et al., Nature 382, 334 (1996).

[10] P.C. Hendry et al, Nature 368, 315 (1994).

[11] M.E. Dodd et al., Phys. Rev. Lett. 81, 3703 (1998), J. Low Temp. Physics 15, 89 (1999).

[12] R. Carmi and E. Polturak, Phys. Rev. B 60, 7595 (1999).

[13] N. Martucciello, J. Mygind, V.P. Koshelets, A.V. Shchukin, L.V. Filippenko and R. Monaco, Phys. Rev. B57, 5444 (1998).

[14] R.J. Rivers, Phys. Rev. Lett 84, 1248 (2000).

[15] G. Karra and R.J. Rivers, Phys. Rev. Lett. 81, 3707 (1998).

[16] R. Carmi, E. Polturak, and G. Koren, Phys. Rev. Lett. 84, 4966 (2000).

[17] M. Hindmarsh and A.Rajantie, Phys. Rev. Lett.85, 4660 (2000); A. Rajantie, Journal of Low Temperature Physics 124, 5 (2001).

[18] S. Casado, W. González-Viñas, H. Mancini and S. Boccaletti, Phys. Rev. E63, 057301 (2001).

[19] S. Ducci, P.L. Ramazza, W. González-Viñas, and F.T. Arecchi, Phys. Rev. Lett. 83, 5210 (1999).

[20] L. V. Filippenko, S. V. Shitov, P. N. Dmitriev, A. B. Ermakov, V. P.Koshelets, and J. R. Gao, IEEE Trans. Appl. Supercond 11, 816-819 (2001).

[21] D.J. Thouless, Phys.Rev. 117, 1256 (1960).

[22] R. Monaco, J. Mygind and R.J. Rivers, in preparation.

\section{FIGURE CAPTIONS}

Fig.1 Sketch (dimensions are not to scale) of the cryogenic insert developed to perform the junction thermal cycles with a time scale changing over a broad range.The JTJs are fabricated within the chip (shown endways), to which the surface mounted resistor (SMR) is attached. The whole is surrounded with liquid He.

Fig.2 Time dependence of the junction temperature during a "slow" thermal cycle from $10 \mathrm{~K}$ to $4.2 \mathrm{~K}$, as inferred from the junction gap voltage. Only below the horizontal dashed line does the continuous curve describe the temperature. The thick dashed line is the best fitting curve to (2), to be extrapolated to $T_{c}$.

Fig.3 Log-log plot of the measured probability $P_{1}$ to trap one fluxon versus the quenching time $\tau_{Q}$. Each point corresponds to 300 thermal cycles. The solid line (slope $b=0.27$ ) is the best linear fit, in good agreement with the 0.25 value expected for symmetric JTJs. Errors in $\tau_{Q}$ are a few per cent, and systematic errors in $P_{1}$ due to the neglect of fluxonantifluxon pairs are $O\left(P_{1}^{2}\right)$, again a few percent. 